Check for updates

Cite this: RSC Adv., 2018, 8, 30755

\title{
Thermal tuning of graphene-embedded waveguide filters based on the polymer-silica hybrid structure
}

\author{
Yue Cao, Baizhu Lin, Yue Sun, Xinchi Che, Yunji Yi, D * Fei Wang and Daming Zhang
}

Graphene-embedded waveguide filters have been widely used in the areas of polarization and mode filtering because of their characteristics of easy fabrication, high integration, and high extinction ratio. In this article, we propose thermal tuning filters based on a graphene-embedded polymer-silica hybrid waveguide. Compared to previously reported filters, this device can realize the efficient adjustment of the relative position between the optical field and graphene layer by thermal tuning. Consequently, the polarization and mode filtering properties of the filter can be adjusted by thermal tuning. This highefficiency tuning characteristic is due to the opposite thermo-optic coefficient of the polymer and silica material. Furthermore, a layer with a low refractive index is embedded in the polymer-silica hybrid core to increase the tuning efficiency. The optical absorption, mode properties, and thermal field distributions were simulated. It was found that such single-mode filters could realize $E_{11}^{x}$-pass or $E_{11}^{x}$-stop selection, and the attenuation variation $(\Delta \alpha)$ was optimized to $32.20 \mathrm{~dB} \mathrm{~cm}^{-1}$ ( $E_{11}^{\times}$mode) using the top electrode and air trench structure with $\Delta T=10 \mathrm{~K}$ and $P=48.39 \mathrm{~mW}$ in the single-mode waveguide. For the multimode waveguide filters, the attenuation variation $(\Delta \alpha)$ of the $E_{m n}^{x}$ modes $\left(E_{11}^{x}, E_{12}^{x}\right.$, and $\left.E_{21}^{x}\right)$ was also calculated. Such thermal tuning filters were found to be compatible with chemical potential regulation graphene modulators and filters, and they can be applied to broadband photonic integrated circuits and mode division multiplexing systems.

Received 16th July 2018

Accepted 28th August 2018

DOI: $10.1039 / c 8 r a 06038 j$

rsc.li/rsc-advances
According to devices using this material, several GBMFs have recently been proposed including inorganic GBMFs, polymer GBMFs, and silica-polymer hybrid GBMFs. Cheng et al. presented a graphene-on-silicon waveguide and achieved a $7.7 \mathrm{~dB}$ attenuation for the TM mode. ${ }^{14} \mathrm{~A}$ polymer mode extinction filter with air cladding obtained an attenuation of $20.7 \mathrm{~dB}$ for TMpolarized light. ${ }^{15}$ In addition, a broadband graphene/glass hybrid waveguide with PMMA cladding achieved an extinction ratio of $27 \mathrm{~dB}$ for TM-polarized light. ${ }^{16}$ These GBMFs can realize the attenuation of only one polarization state of the base mode in the waveguide with high extinction ratio properties. However, the hybrid structure has not made full use of the advantages of the hybrid integration.

In addition, according to the change in the chemical potential of graphene, GBMFs are divided into electro-optic GBMFs and non-electro-optic GBMFs. For graphene electroabsorption modulator on silicon, a graphene absorption loss of $0.11 \mathrm{~dB} \mu \mathrm{m}^{-1}$ and $0.06 \mathrm{~dB} \mu \mathrm{m}^{-1}$ were extracted for the TM and TE modes respectively. ${ }^{17}$ Non-electro-optic GBMFs with two graphene layers sandwiched between $\mathrm{Si}_{3} \mathrm{~N}_{4}$ and silicon layers achieved an extinction ratio higher than $40 \mathrm{~dB} \cdot{ }^{15}$ The devices reported above have achieved high extinction ratios for the base mode of the waveguide. However, polarization states of GBMFs between TE-pass and TM-pass cannot be switched by changing the chemical potential. Thus, it has been proposed that the polarization states are attributed to waveguide parameters such 
as the device dimension and refractive index. Thus, different structures of non-electro-optic GBMFs are shown to be essential for the development of mode filters. ${ }^{18}$

Moreover, in the area of MDM, a mode filter reportedly embedded in different positions and lengths of graphene in the waveguide core realized the attenuation of different modes in a multimode waveguide. This mode filter with a graphene layer placed at the center of the waveguide core realizes the attenuation of the $E_{11}^{\mathrm{x}}$ mode and $\mathrm{E}_{21}^{\mathrm{x}}$ mode. When the graphene film is placed at the location where the $\mathrm{E}_{13}^{\mathrm{x}}$ mode is zero, the filter realizes the attenuation of the $\mathrm{E}_{11}^{\mathrm{x}}, \mathrm{E}_{21}^{\mathrm{x}}$, and $\mathrm{E}_{12}^{\mathrm{x}}$ modes. In addition, when a narrow graphene film is placed at the center of the waveguide core, the filter attenuates the $E_{11}^{x}$ mode. $^{3}$ However, fixed graphene-based waveguide structures have fixed mode attenuation characteristics.

In this paper, a thermal tuning filter based on a grapheneembedded polymer-silica hybrid waveguide is presented. The position of the light field of this filter structure can be regulated by varying the electrode temperature. Meanwhile, the relative position between the graphene and optical field can be regulated, which results in the change in the absorption of different modes. Because of the opposite thermo-optic coefficient of the polymer and silica material, the optical field can be regulated with a high efficiency. Two structures of the optimized thermal tuning hybrid filters with high extinction ratios have been obtained with a top electrode and side electrodes.

\section{Design and simulation}

Our proposed filters are based on the concept of embedding a graphene layer in a waveguide to generate strong absorption of the tangential electric field, $E_{\|}$, which is dominantly composed of the $E_{x}$ direction for $\mathrm{E}_{m n}^{\mathrm{x}}$ modes and $E_{z}$ direction for $\mathrm{E}_{m n}^{\mathrm{y}}$ modes. The $\mathrm{E}_{m n}^{\mathrm{x}}$ and $\mathrm{E}_{m n}^{\mathrm{y}}$ modes denote the $x$ and $y$ directions of the major electric-field component, respectively, and $m$ and $n$ denote the numbers of peaks in the major electric-field distribution. We designed a graphene layer embedded in a polymer core waveguide, which adheres to a silica waveguide. The isotropic model of the graphene was used in the simulation for $\mathrm{E}_{m n}^{\mathrm{x}}$ modes. ${ }^{19}$ Before thermal tuning, the center position of the $\mathrm{E}_{11}^{\mathrm{x}}$-mode light field was designed to be located in the graphene layer. At this position, the graphene exhibits the maximum light-graphene interaction with the $\mathrm{E}_{11}^{\mathrm{x}}$ mode. While the graphene layer should not induce much loss to the $\mathrm{E}_{m n}^{\mathrm{y}}$ modes. Also, the polymer-silica vertical hybrid waveguide we designed will ensure the best tuning effect because of the opposite thermo-optic coefficient of the polymer and inorganic material. If we raise the working temperature, the refractive index of the polymer should decrease while the silica will increase, leading to a change in the distribution of the light field of the $\mathrm{E}_{m n}^{\mathrm{x}}$ polarization state. The redistribution of the $\mathrm{E}_{m n}^{\mathrm{x}}$ optical fields will realize mode attenuation in the singlemode waveguide and multimode waveguide, respectively. For the heating electrode structure of the top electrode and side electrode, the top electrode with an air trench structure (structure 1) was designed to realize $\mathrm{E}_{11}^{\mathrm{x}}$ mode attenuation and the $\mathrm{E}_{11}^{\mathrm{y}}$-pass waveguide. In addition, the side electrode structure (structure 2) was designed to take advantage of the high thermal conductivity of graphene to reduce the power consumption. ${ }^{20}$

\section{Device structure and theoretical analysis}

The absorption of the light in waveguide is mainly related to the imaginary part of the refractive index of graphene. The graphene effective permittivity $\varepsilon_{\mathrm{g}}\left(=n_{\mathrm{g}}{ }^{2}\right)$ can be calculated from the conductivity $\sigma$ :

$$
\varepsilon_{\mathrm{g}}(\omega)=\varepsilon_{\infty}+\frac{i \sigma}{\omega \varepsilon_{0} d_{\mathrm{g}}}
$$

where $\varepsilon_{\infty}=1$ is the graphene background permittivity, $\omega=$ $1.215 \times 10^{15} \mathrm{rad} \mathrm{s}^{-1}$ is the angular frequency of the light, and $\varepsilon_{0}$ is the vacuum permittivity.

The graphene conductivity $\sigma$ is affected by the temperature, graphene electronic relaxation time, optical angular frequency, and graphene Fermi level. The parameters of graphene conductivity, including intraband conductivity and interband conductivity, can be calculated using the Kubo formalism:

$$
\begin{gathered}
\sigma=\sigma_{\text {intra }}+\sigma_{\text {inter }} \\
\sigma(\omega)=\frac{\mathrm{i} e^{2} u_{\mathrm{c}}}{\pi \hbar^{2}\left(\omega+\mathrm{i} \tau^{-1}\right)}+\frac{\mathrm{i} e^{2}}{4 \pi \hbar} \ln \left[\frac{2\left|u_{\mathrm{c}}\right|-\left(\omega+\mathrm{i} \tau^{-1}\right) \hbar}{2\left|u_{\mathrm{c}}\right|+\left(\omega+\mathrm{i} \tau^{-1}\right) \hbar}\right],
\end{gathered}
$$

where $u_{\mathrm{c}}=0.1 \mathrm{eV}$ is the chemical potential, $\hbar$ is the reduced Planck's constant, and $\tau=5 \times 10^{-13} \mathrm{~s}$ is the relaxation time. The electrode position, waveguide structure, and geometric parameters including the waveguide height, waveguide width, and refractive index are discussed using the FD-BPM method in the single-mode waveguide and multimode waveguide.

Device structure 1 (top electrode structure with an air trench) and structure 2 (side electrode structure without air trench) in the three-dimension and cross-sectional directions are illustrated in Fig. 1. The device consists of a silica substrate, polymer cladding, polymer-silica hybrid core layer, graphene strip embedded in the polymer material, and sheet layer with a low refractive index embedded between the hybrid waveguide core. The dimensions of the polymer-silica hybrid layer are $a=b=6 \mu \mathrm{m}$. The parameters $d_{\mathrm{e}}=0.1 \mu \mathrm{m}, d_{\mathrm{g}}=$ $0.00034 \mu \mathrm{m}$, and $d_{\mathrm{p}}=0.1 \mu \mathrm{m}$ indicate the thickness of the electrode, graphene layer, and sheet layer, respectively. $n_{1}=$ $1.467, n_{2}=1.466$, and $n_{4}=1.455$ are the refractive indices of the polymer layer, silica layer, and silica cladding layer, respectively. The height of the polymer waveguide $h$ and the refractive index of polymer cladding $n_{3}$ are variables to adjust the position of the light field in the waveguide. The calculated imaginary and real part of the refractive index is 2.79 and 2.97 respectively.

\section{Thermal field simulation}

The position of the electrode will influence the heating efficiency of the polymer-silica hybrid waveguide. Fig. 2 presents the thermal field distribution of the top electrode with $w=10$ $\mu \mathrm{m}$ (an air trench is etched to increase the electrode heating efficiency) and side electrode structure with $w=6 \mu \mathrm{m}$. The temperature of the central polymer layer is $297.26 \mathrm{~K}$ 


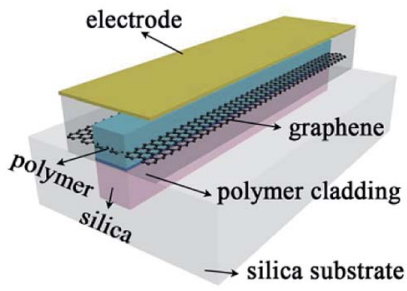

(a)

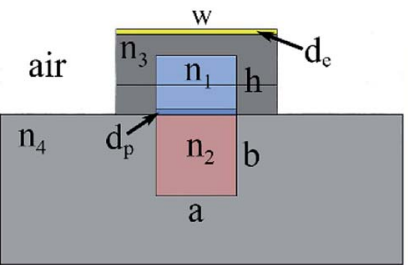

(c)

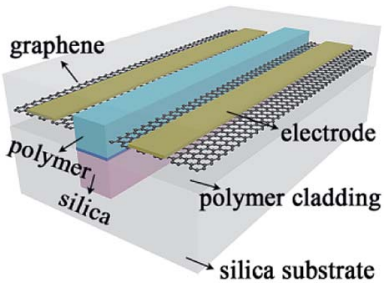

(b)

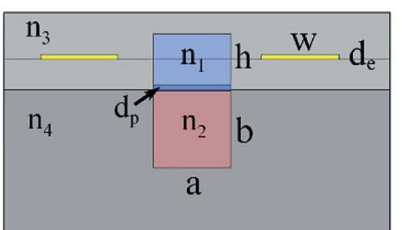

(d)
Fig. 1 Three-dimensional structures and two-dimensional crosssectional structures of polymer-graphene-silica hybrid waveguide with structure ( $a$ and c) 1 and (b and d) 2, respectively.

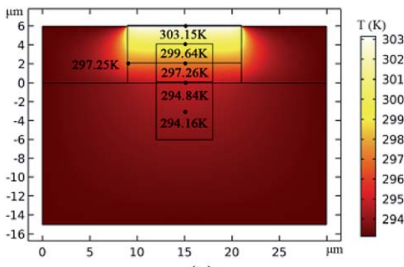

(a)

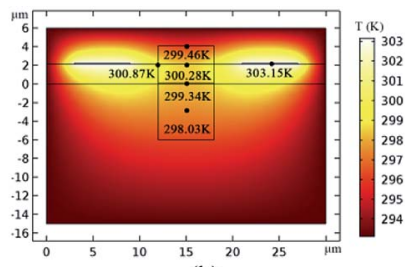

(b)
Fig. 2 Thermal field distribution for (a) top electrode structure with $w$ $=10 \mu \mathrm{m}$ and (b) side electrode structure with $w=6 \mu \mathrm{m}$.

(structure 1) and 300.28 $\mathrm{K}$ (structure 2), which indicates that the side electrode structure has a higher heating efficiency than the top electrode structure.

\section{Single-mode simulation}

In order to realize a low-power-consumption device, a sheet $\left(d_{\mathrm{p}}\right.$ $=0.1 \mu \mathrm{m}$ ) with a low refractive index was embedded in the hybrid waveguide, which is a benefit for the light field moving away from the graphene layer, reducing the graphene absorption in the hybrid waveguide. The imaginary part of the effective refractive index has been calculated by COMSOL with a wavelength of $1550 \mathrm{~nm}$. The thermo-optical coefficients of polymer and silica are $-3.0 \times 10^{-4}$ and $1.28 \times 10^{-5}$ respectively. ${ }^{21}$

Without the sheet layer, the optical field distribution of the hybrid waveguide with electrode temperatures of 293.15 and $303.15 \mathrm{~K}$ is presented in Fig. 3(a) and (b), respectively. The simulated light absorption is 94.78 and $67.15 \mathrm{~dB} \mathrm{~cm}^{-1}$ at electrode temperatures of 293.18 and $303.15 \mathrm{~K}$, respectively, and the $\mathrm{E}_{11}^{\mathrm{x}}$-polarized light still cannot pass through the waveguide when the heating electrode increases by $10 \mathrm{~K}$ with electrode heating power $(P)$ of $48.39 \mathrm{~mW} .^{22}$

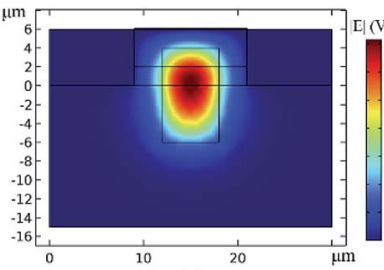

(a)

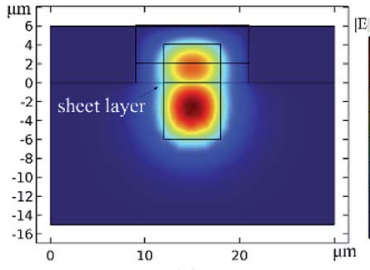

(c)

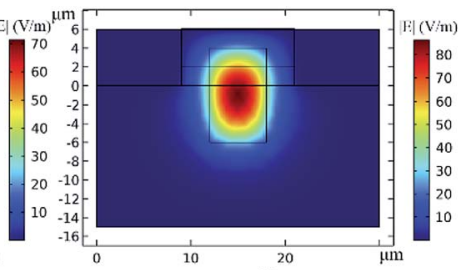

(b)

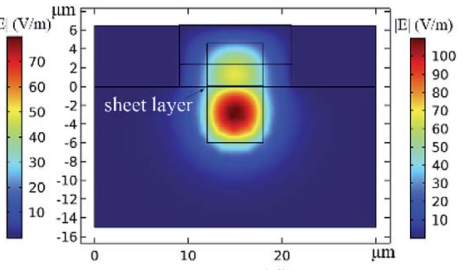

(d)
Fig. 3 Optical field distribution of $E_{11}^{x}$-polarized light without and with a sheet layer embedded in waveguide core for $T=$ (a and c) 293.15 and (b and d) $303.15 \mathrm{~K}$ (structure 1), respectively (electrode heating power $(P)=48.39 \mathrm{~mW})$.

By varying the height of the polymer material layer and the refractive index of the polymer cladding, a single-mode waveguide was obtained. With a sheet layer embedded in the hybrid waveguide, Fig. 3(c) and (d) present the light field distribution at temperatures of 293.15 and $303.15 \mathrm{~K}$, respectively (structure 1).

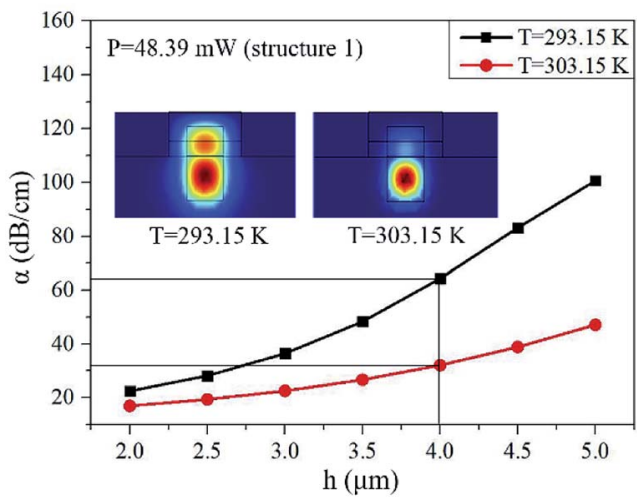

(a)

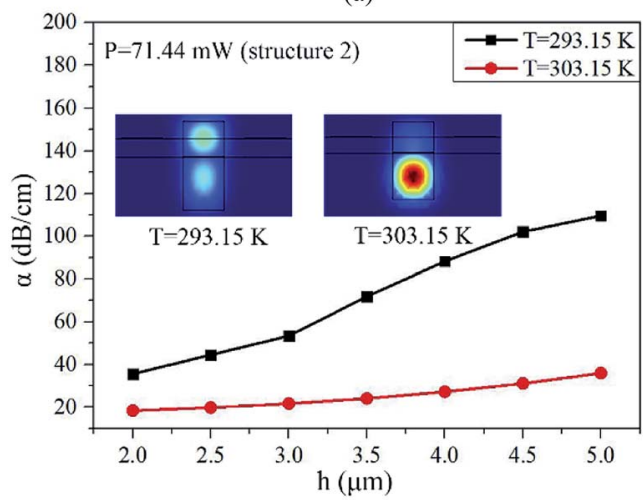

(b)

Fig. 4 Light field distribution and attenuation of $E_{11}^{x}$ mode with different height of polymer layer and electrode temperature $\left(n_{3}=\right.$ 1.460) for (a) structure 1 and (b) structure 2, respectively. 


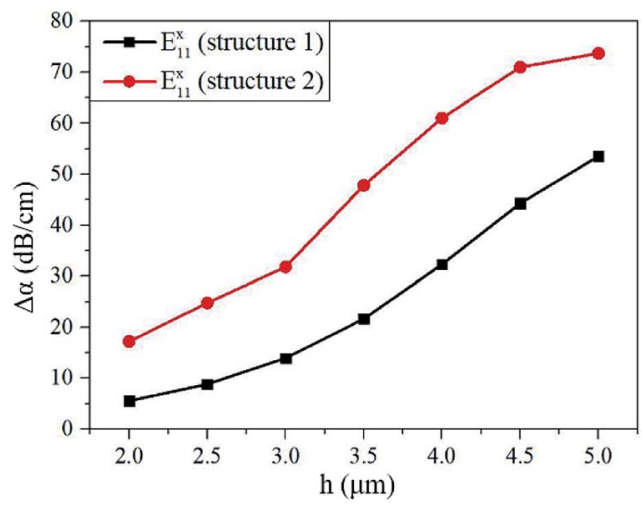

(a)

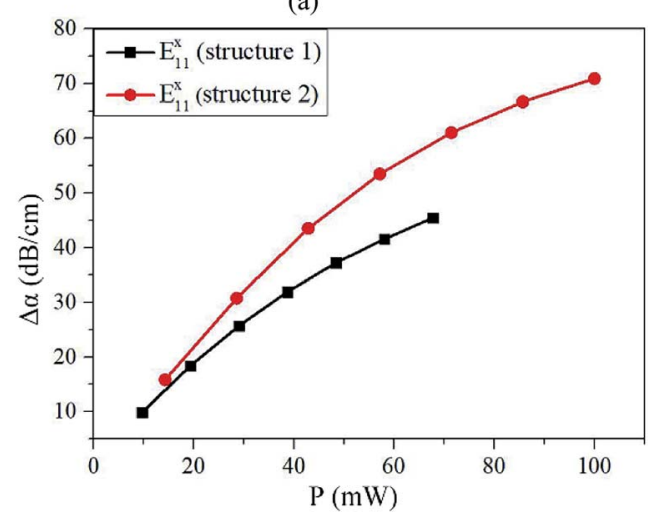

(b)

Fig. 5 Curves of the polymer layer height and attenuation variation $(\Delta \alpha)$ for structure 1 and structure 2 as a function of (a) polymer waveguide height $h$ and (b) electrode heating power $P$, respectively $\left(n_{3}\right.$ $=1.460$ ).

The light field distribution and attenuation of $E_{11}^{\mathrm{x}}$ mode as a function of $h$ with electrode temperature of $293.15 \mathrm{~K}$ and 303.15 K for structure 1 are shown in Fig. 4(a). From the light field distribution, the graphene layer exhibits a significant interaction with the $\mathrm{E}_{11}^{\mathrm{x}}$ mode in the $x$-direction at the temperature of $293.15 \mathrm{~K}$ ( $\mathrm{E}_{11}^{\mathrm{x}}$-stop waveguides). When the electrode heating power increases by $48.39 \mathrm{~mW}$, the light field distribution is more concentrated in the silica layer, reducing the interaction between graphene and the $E_{11}^{\mathrm{x}}$ modes ( $E_{11}^{\mathrm{x}}$-pass waveguides). Thus, it is a benefit for increasing the attenuation of the $E_{11}^{x}$ mode at $293.15 \mathrm{~K}$ if the height of the polymer layer increases. However, it will also increase the light absorption of the graphene layer at $303.15 \mathrm{~K}$. For example, the attenuation of the $E_{11}^{\mathrm{x}}$ mode increases to $47.12 \mathrm{~dB} \mathrm{~cm}^{-1}$ at an electrode temperature of $303.15 \mathrm{~K}$, which cannot realize the $\mathrm{E}_{11}^{\mathrm{x}}$-pass waveguide.

Fig. 4(b) presents the light field distribution and the attenuation curves with $h=4 \mu \mathrm{m}, n_{3}=1.460$ and $P=71.44 \mathrm{~mW}$ for structure 2. Because of polarized light effect by the surface plasmon polaritons (SPPs) of the side electrode placed on the graphene layer, the attenuation of the $E_{11}^{\mathrm{x}}$ mode increases by $23.85 \mathrm{~dB} \mathrm{~cm}^{-1}$, compared to structure 1 at $293.15 \mathrm{~K}$.

Based on the above discussion, the attenuation variation $(\Delta \alpha$ $\left.=\alpha_{T}(303.15 \mathrm{~K})-\alpha_{T}(293.15 \mathrm{~K})\right)$ has been calculated with $h=4$ $\mu \mathrm{m}$ and $n_{3}=1.460$, as shown in Fig. 5. For structure 1, the $\Delta \alpha$ of

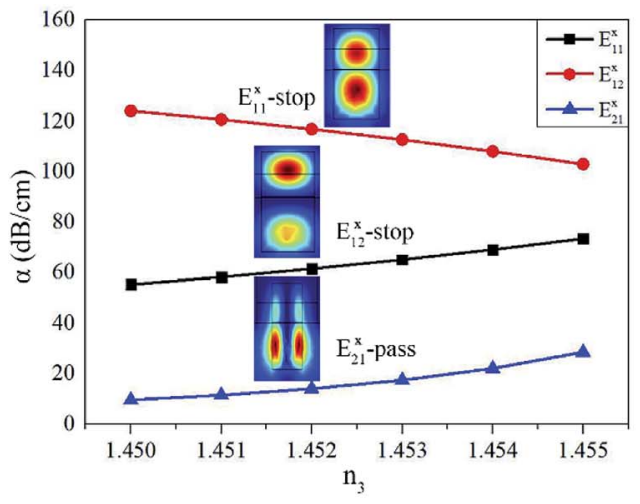

(a)

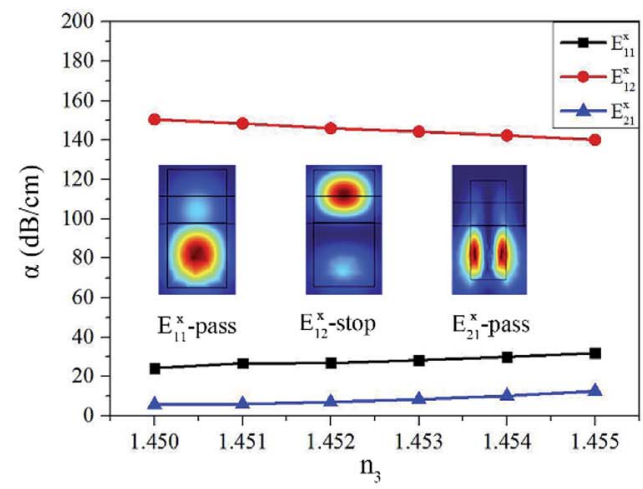

(b)

Fig. 6 Light field distribution and the attenuation of $E_{11}^{x}$ mode, $E_{12}^{x}$ mode and $E_{21}^{x}$ mode with different polymer cladding $n_{3}(h=5 \mu \mathrm{m})$ at (a) $T=293.15 \mathrm{~K}$ and (b) $T=303.15 \mathrm{~K}$.

the $\mathrm{E}_{11}^{\mathrm{x}}$ mode is $32.30 \mathrm{~dB} \mathrm{~cm}^{-1}$, realizing the regulation of the $\mathrm{E}_{11}^{\mathrm{x}}$ mode by heating the electrode. For structure 2, the $\Delta \alpha$ of the $\mathrm{E}_{11}^{\mathrm{x}}$ mode increases up to $61.06 \mathrm{~dB} \mathrm{~cm}^{-1}$, realizing the efficient regulation of the $E_{11}^{x}$ mode waveguide by heating the side electrode. This can be contributed to the effect of the electrode SPPs and the large heating efficiency (discussed later in the article) for the side electrode structure. Fig. 5(b) illustrates the $\mathrm{E}_{11}^{\mathrm{x}}$ mode in structure 1 and structure 2 with different electrode heating power. It is indicated that the device is sensitive to the change in electrode temperature.

\section{Multimode simulation}

By increasing the height of the polymer layer or decreasing the refractive index of the polymer cladding, a hybrid multimode waveguide can be obtained. Fig. 6 depicts the relationships between the cladding refractive index and $\alpha$ of the multimode waveguide ( $\mathrm{E}_{11}^{\mathrm{x}}, \mathrm{E}_{12}^{\mathrm{x}}$ and $\mathrm{E}_{21}^{\mathrm{x}}$ modes $)$ at $T=293.15 \mathrm{~K}$ and $T=$ $303.15 \mathrm{~K}(h=5 \mu \mathrm{m}$ and $P=43.86 \mathrm{~mW})$ for device structure 1 . When the electrode temperature increases by $10 \mathrm{~K}$, the $\mathrm{E}_{11^{\mathrm{x}}}^{\mathrm{x}}$ stop waveguide, $E_{12}^{\mathrm{x}}$-stop waveguide and $\mathrm{E}_{21}^{\mathrm{x}}$-pass waveguide have been realized. When $T=303.15 \mathrm{~K}$, the $\mathrm{E}_{11}^{\mathrm{x}}$-pass waveguide, $\mathrm{E}_{12}^{\mathrm{x}}$-stop waveguide and $\mathrm{E}_{21}^{\mathrm{x}}$-pass waveguide have been realized.

Fig. 7 shows the attenuation variation $(\Delta \alpha)$ of $E_{11}^{x}, E_{12}^{x}$, and $\mathrm{E}_{21}^{\mathrm{x}}$ modes as function of cladding refractive index for structure $1(h=5 \mu \mathrm{m} ; P=43.86 \mathrm{~mW})$. The regulation of the modes can 


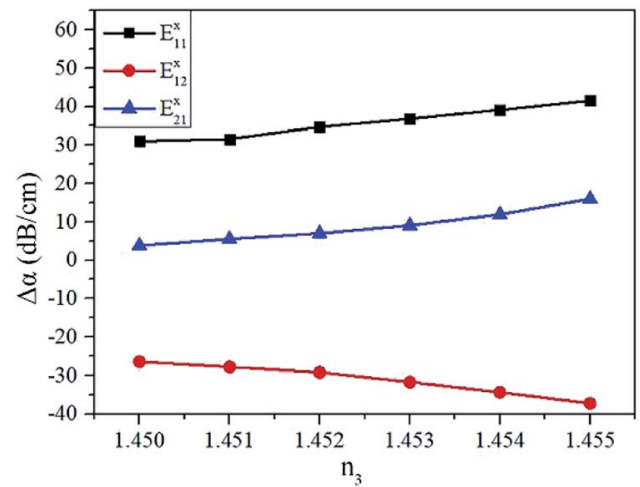

Fig. 7 Relationships between cladding refractive index $\left(n_{3}\right)$ and attenuation variation $(\Delta \alpha)$ of $E_{11}^{x}, E_{12}^{x}$, and $E_{21}^{x}$ modes $(h=5 \mu \mathrm{m} ; P=$ $43.86 \mathrm{~mW}$; structure 1).

also be calculated with the attenuation variation $(\Delta \alpha)$ from 35.70 to $47.84 \mathrm{~dB} \mathrm{~cm}{ }^{-1}$ ( $\mathrm{E}_{11}^{\mathrm{x}}$ mode), from 42.89 to -30.47 $\mathrm{dB} \mathrm{cm}^{-1}$ ( $\mathrm{E}_{12}^{\mathrm{x}}$ mode), from 4.42 to $18.45 \mathrm{~dB} \mathrm{~cm}^{-1}$ ( $\mathrm{E}_{21}^{\mathrm{x}}$ mode). The optical transmission states (mode-pass or mode-stop) of the multimode-polarized light depend on the device dimension and refractive index. Thus, the optical transmission state for the same polarized light may change with different cladding refractive indices and device dimensions.

We expect that the multimode-polarized light can be adjusted with different conditions of the refractive index by the heating electrode, realizing multimode thermal tuning filters. However, because the multimode-polarized light is affected significantly by SPPs of the side electrode structure, the transmission state in structure 2 is more complex than that of structure 1 with different device dimensions and refractive indices, which doesn't benefit the adjustment of the multimode-polarized light by changing the temperature of the side electrode.

\section{Conclusions}

In summary, we have presented ultra-broadband thermal tuning filters based on a graphene embedded polymer-silica hybrid waveguide. This device can realize the efficient adjustment of the relative position between the optical field and graphene layer by thermal tuning. For a single-mode waveguide, we achieved the regulation of the $\mathrm{E}_{11}^{\mathrm{x}}$ mode with $\Delta \alpha=32.20$ $\mathrm{dB} \mathrm{cm}{ }^{-1}$ and the $E_{11}^{\mathrm{x}}$-pass waveguide for structure 1 . We also obtained low-power-consumption devices with a high $\Delta \alpha$ of $61.06 \mathrm{~dB} \mathrm{~cm}^{-1}$ ( $\mathrm{E}_{11}^{\mathrm{x}}$ mode) for structure 2 in the single-mode waveguide $(\Delta T=10 \mathrm{~K})$. Moreover, the side electrode structure had a higher heating efficiency than the top electrode structure. For the multimode waveguide in structure 1 , the multimodepolarized light can be regulated by increasing the electrode temperature. The regulation of the modes was analyzed with the attenuation variation $(\Delta \alpha)$ from 35.70 to $47.84 \mathrm{~dB} \mathrm{~cm}^{-1}$ ( $\mathrm{E}_{11}^{\mathrm{x}}$ mode), from -42.89 to $-30.47 \mathrm{~dB} \mathrm{~cm}^{-1}$ ( $\mathrm{E}_{12}^{\mathrm{x}}$ mode), from 4.42 to $18.45 \mathrm{~dB} \mathrm{~cm}^{-1}$ ( $\mathrm{E}_{21}^{\mathrm{x}}$ mode). However, the power consumption is restricted by single-layer graphene structure and large device dimension because of the photolithographic process. The graphene-based filter with a top electrode and air trench structure (structure 1) can be applied to many photonic devices such as thermo-optical switches, MDM systems, and variable optical attenuators. In addition, the graphene-based filter with a side electrode and without an air trench structure (structure 2) has the potential for compatibility with thermooptical devices and electro-optic devices.

\section{Funding}

National Natural Science Foundation of China (NSFC) (61605057, 61475061, 61575076); Jilin Provincial Industrial Innovation Special Fund Project (2016C019); Excellent Youth Foundation of Jilin Province (20170520158JH).

\section{Conflicts of interest}

There are no conflicts to declare.

\section{References}

$1 \mathrm{~S}$. J. Koester and M. Li, High-speed waveguide-coupled graphene-on-graphene optical modulators, Appl. Phys., 2012, 100, 171107.

2 Q. Jin, X. B. Li, J. F. Chen and S. M. Gao, Ultra-broadband nonlinearity enhancement based on a novel graphenesilicon hybrid waveguide: Structure design and theoretical analysis, Sci. Rep., 2017, 7, 12290.

3 Z. Chang and K. S. Chiang, Ultra-broadband mode filters based on graphene-embedded waveguides, Opt. Lett., 2017, 42, 3868-3871.

4 M. Liu, X. B. Yin, E. Ulin-Avila, B. S. Geng, T. Zentgraf, L. Ju, F. Wang and X. Zhang, A graphene-based broadband optical modulator, Nature, 2011, 474, 64-67.

5 L. Z. Yang, T. Hu, R. Hao, C. Qiu, C. Xu, H. Yu, Y. Xu, X. Q. Jiang, Y. B. Li and J. Y. Yang, Low-chirp highextinction-ratio modulator based on graphene-silicon waveguide, Opt. Lett., 2013, 38, 2512-2515.

6 A. Pospischil, M. Humer, M. M. Furchi, D. Bachmann, R. Guider, T. Fromherz and T. Mueller, CMOS-compatible graphene photodetector covering all optical communication bands, Nat. Photonics, 2013, 7, 892-896.

7 A. Andryieuski and A. V. Lavrinenko, Graphene metamaterials based tunable terahertz absorber: effective surface conductivity approach, Opt. Express, 2013, 21, 9144-9155.

8 X. Wang, Z. Cheng, K. Xu, H. K. Tsang and J.-B. Xu, Highresponsivity graphene/silicon-heterostructure waveguide photodetectors, Nat. Photonics, 2013, 7, 888-891.

9 J. Ma, W. Jin, H. L. Ho and J. Y. Dai, High-sensitivity fiber-tip pressure sensor with graphene diaphragm, Opt. Lett., 2012, 37, 2493-2495.

10 C. Z. Tan, L. Cao and T. B. Wang, Polarized beam splitting by total internal reflection in $\alpha$-quartz, Nucl. Instrum. Methods Phys. Res., Sect. B, 2005, 239, 267. 
11 B. A. Dorin and W. N. Ye, Two-mode division multiplexing in a silicon-on-insulator ring resonator, Opt. Express, 2014, 22, 4547-4558.

$12 \mathrm{X}$. Guan, Y. Ding and L. H. Frandsen, Ultra-compact broadband higher order-mode pass filter fabricated in a silicon waveguide for multimode photonics, Opt. Lett., 2015, 40, 3893-3896.

13 P. Xing, K. J. A. Ooi and D. T. H. Tan, Ultra-broadband and compact graphene-on-silicon integrated waveguide mode filters, Sci. Rep., 2018, 8, 1-9.

14 Z. Cheng, H. K. Tsang, X. Wang, X. Chen, K. Xu, and J.-B. Xu, Polarization dependent loss of graphene-on-silicon waveguides, in Proceedings of 2013 IEEE Photonics Conference, IEEE, 2013, pp. 460-461.

$15 \mathrm{X}$. Hu and J. Wang, Ultrabroadband compact graphenesilicon TM-pass polarizer, IEEE Photonics J., 2017, 9, 7101310.

16 C. Pei, L. Yang, G. Wang, Y. Wang, X. Jiang, Y. Hao, Y. Li and J. Yang, Broadband graphene/glass hybrid waveguide polarizer, IEEE Photonics Technol. Lett., 2015, 27, 927-930.

17 Y. T. Hu, M. Pantouvaki, J. Van Campenhout., S. Brems, I. Asselberghs, C. Huyghebaert, P. Absil and D. Van
Thourhout, Broadband $10 \mathrm{~Gb} / \mathrm{s}$ operation of graphene electro-absorption modulator on silicon, Laser Photonics Rev., 2016, 10, 307-316.

18 R. de Oliveira and C. de Matos, Graphene Based Waveguide Polarizers: In-Depth Physical Analysis and Relevant Parameters, Sci. Rep., 2015, 5, 16949.

19 Z. Chang and K. S. Chiang, Experimental verification of optical models of graphene with multimode slab waveguides, Opt. Lett., 2016, 41, 2129.

20 Y. Sun, Y. Cao, Y. Yi, L. Tian, Y. Zheng, J. Zheng, F. Wang and D. Zhang, A low-power consumption MZI thermal optical switch with a graphene-assisted heating layer and air trench, RSC Adv., 2017, 7, 39922-39927.

21 Z. Zhiyi, P. Zhao, L. Peng and S. Fengguo, Thermo-optic coefficients of polymers for optical waveguide applications, Polymer, 2006, 47, 4893-4896.

22 L. Yu-Fen, W. Fei, W. Xi-Bin, S. Xiao-Qiang, S. Jian, Gu. Hong-Jun, C. Chang-Ming and Z. Da-Ming, Thermal field analysis of polymer/silica hybrid waveguide thermo-optic switch, Elsevier B.V, 2015, vol. 356, pp. 79-83. 